\title{
Strongly anisotropic spin relaxation revealed by resonant spin amplification in (110) GaAs quantum wells
}

\author{
M. Griesbeck, ${ }^{1}$ M. M. Glazov,,${ }^{2 *}$ E. Ya. Sherman, ${ }^{3}$ D. Schuh,${ }^{1}$ W. Wegscheider,${ }^{4}$ C. Schüller, ${ }^{1}$ and T. Korn ${ }^{1, \dagger}$ \\ ${ }^{1}$ Institut für Experimentelle und Angewandte Physik, Universität Regensburg, D-93040 Regensburg, Germany \\ ${ }^{2}$ Ioffe Physical-Technical Institute, Russian Academy of Sciences, 194021 St. Petersburg, Russia \\ ${ }^{3}$ Department of Physical Chemistry, The University of the Basque Country UPVIEHU, 48080 Bilbao, Spain and \\ IKERBASQUE Basque Foundation for Science, Alameda Urquijo 36-5, 48011 Bilbao, Bizkaia, Spain \\ ${ }^{4}$ Solid State Physics Laboratory, ETH Zurich, 8093 Zurich, Switzerland
}

(Received 19 December 2011; published 27 February 2012)

\begin{abstract}
We have studied spin dephasing in a high-mobility two-dimensional electron system confined in a GaAs/AlGaAs quantum well grown in the [110] direction, using the resonant spin amplification (RSA) technique. From the characteristic shape of the RSA spectra, we are able to extract the spin dephasing times (SDTs) for electron spins aligned along the growth direction or within the sample plane, as well as the $g$ factor. We observe a strong anisotropy in the spin dephasing times. While the in-plane SDT remains almost constant as the temperature is varied between 4 and $50 \mathrm{~K}$, the out-of-plane SDT shows a dramatic increase at a temperature of about $25 \mathrm{~K}$ and reaches values of about $100 \mathrm{~ns}$. The SDTs at $4 \mathrm{~K}$ can be further increased by additional, weak above-barrier illumination. The origin of this unexpected behavior is discussed. The SDT enhancement is attributed to the redistribution of charge carriers between the electron gas and remote donors.
\end{abstract}

DOI: 10.1103/PhysRevB.85.085313

PACS number(s): 73.61.Ey, 75.40.Gb, 85.75.-d

\section{INTRODUCTION}

Two-dimensional electron systems (2DESs) based on the GaAs/AlGaAs materials are promising candidates for semiconductor spintronics ${ }^{1,2}$ devices. They offer very high electron mobilities and allow one to manipulate the spin orientation by electric fields ${ }^{3}$ via the Rashba spin-orbit interaction (SOI). ${ }^{4}$ For structures grown along the [110] crystallographic direction, spin dephasing via the Dyakonov-Perel (DP) mechanism ${ }^{5}$ is strongly suppressed for growth-axis-oriented spins, ${ }^{6,7}$ while it remains active for other spin orientations. ${ }^{8}$ A similar anisotropy of the spin dephasing arises in [001]-grown structures for equal strength of Rashba and Dresselhaus fields. ${ }^{9-13}$ For such structures, however, the suppression of the DP mechanism occurs along one in-plane crystallographic orientation. While all-electrical devices are envisioned for most future semiconductor spintronics applications, optical spectroscopy techniques have proven to be very useful for the study of spin dynamics in direct-gap semiconductor heterostructures, and a variety of techniques, including time-resolved Faraday rotation (TRFR) ${ }^{14}$ Hanle measurements, and spin noise spectroscopy (SNS $)^{15}$ have been developed. A number of experimental groups have studied spin dephasing in various (110)-grown systems. For nominally undoped quantum wells (QWs), growth-axis spin dephasing times (SDTs) of 2-4 ns at room temperature were reported, $, 3,7$ and at low temperatures, using surface acoustic waves to laterally transport optically oriented electrons, SDTs of 18 ns were reached. ${ }^{16}$ The temperature dependence of the SDTs in a (110)-grown 2DES was studied in Ref. 8 using time-resolved photoluminescence, yielding values of the growth-axis SDT between $1.8 \mathrm{~ns}$ at liquid-helium temperature and $6.5 \mathrm{~ns}$ at $120 \mathrm{~K}$. In optical studies of spin dynamics, the use of interband excitation or probing always generates electron-holes pairs, and the optically created holes provide a spin dephasing channel via the Bir-Aronov-Pikus (BAP) mechanism, ${ }^{17}$ hampering the approach to the SDT of an unperturbed system. The largest value for the growth-axis SDT in a (110)-grown 2DES reported so far, $24 \mathrm{~ns},{ }^{18,19}$ was determined by the SNS technique in the limit of weak optical probing of the equilibrium spin dynamics. The large SDT values in (110)-grown 2DES also allowed the observation of hyperfine interaction between nuclei and electron spins in an all-optical nuclear magnetic resonance experiment. $^{20}$

Here, we present time-resolved optical studies of a highmobility (110)-grown 2DES using the resonant spin amplification (RSA) technique, ${ }^{21,22}$ a variation of the TRFR technique, which has been successfully applied to study electron and hole spin dynamics in systems of different dimensionality. ${ }^{22-25} \mathrm{We}$ observe SDTs of about $100 \mathrm{~ns}$ at low temperatures, exceeding the previously reported values for free electrons in a 2DES by almost one order of magnitude. We show that the SDTs extracted from RSA spectra are not limited by the BAP mechanism and use an optical gating technique to control the 2DES carrier density and growth-axis symmetry to reach even higher SDT values.

\section{SAMPLE STRUCTURE AND EXPERIMENTAL METHODS}

Our sample contains a symmetrically $n$-modulation-doped, 30 -nm-wide GaAs QW in which the 2DES resides. It is similar in design to structures introduced by Umansky et al. ${ }^{26}$ Figure 1(a) shows the schematic layer structure of the sample: a total of four $n$-doping layers are deposited in the barrier material left and right of the GaAs QW. While the doping layers far to the left and to the right of the QW mostly serve to give flatband conditions, the two closer doping layers provide the charge carriers for the 2DES. These doping layers are embedded between two 2-nm-thick layers of AlAs, so that some of the dopant electrons occupy the $X$ valley states in the AlAs layers and lead to partial screening of the dopant disorder potential. ${ }^{27} \mathrm{~A}$ sketch of the resulting band structure 

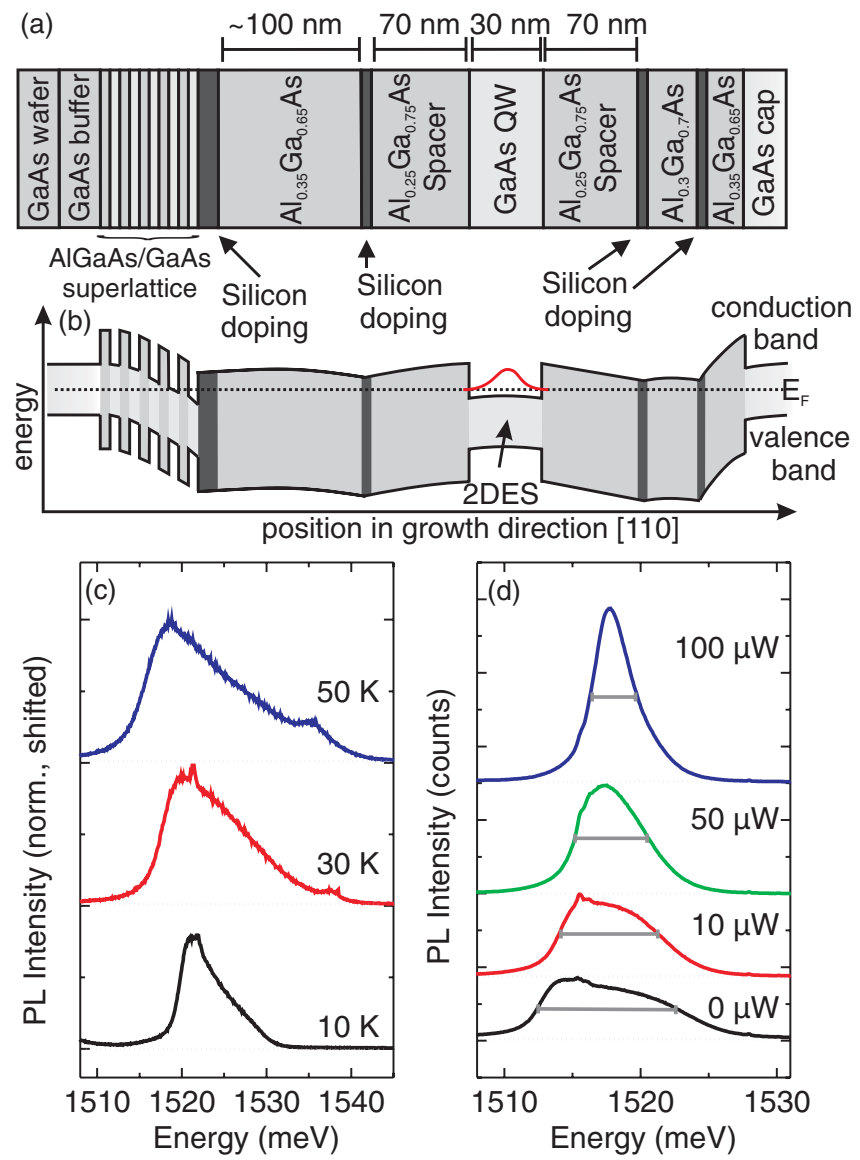

FIG. 1. (Color) (a) Schematic layer structure of the investigated sample. (b) Schematic band structure of the investigated sample. (c) PL traces measured at three different temperatures. (d) PL traces measured at $4 \mathrm{~K}$ for different excitation densities of above-barrier illumination.

is given in Fig. 1(b), showing the well-defined symmetric confinement of the 2DES in the QW. The nominal carrier density $n=2.7 \times 10^{11} \mathrm{~cm}^{-2}$ and mobility $\mu=2.3 \times 10^{6}$ $\mathrm{cm}^{2}(\mathrm{~V} \mathrm{~s})^{-1}$ of our sample were determined at $1.5 \mathrm{~K}$ using magnetotransport measurements. In similar structures grown on (001) substrates, even higher carrier mobilities above $18 \times 10^{6} \mathrm{~cm}^{2}(\mathrm{~V} \mathrm{~s})^{-1}$ were observed at low temperatures, allowing us to study the spin dynamics of electrons on ballistic cyclotron orbits. ${ }^{28,29}$ The sample is mounted in vacuum in a He-flow cryostat during measurements, and the sample temperature is varied between 4 and $50 \mathrm{~K}$. We note that the 2DES electron temperature [extracted from analysis of the photoluminescence (PL) line shape, namely, by fitting the high-energy tail with a Fermi-Dirac distribution function] is higher than the lattice temperature, and remains above $15 \mathrm{~K}$ even for the lowest sample temperatures, as the high mobility of the sample corresponds to very inefficient electron-lattice coupling. We utilize a pulsed Ti:sapphire laser system to excite electrons in the 2DES slightly above the Fermi energy with a circularly polarized pump pulse, and a time-delayed, linearly polarized probe pulse from the same laser is used to detect the growth-axis spin polarization in the 2DES via the spin Kerr effect. The laser pulse length is about $2 \mathrm{ps}$, corresponding to a spectral linewidth of the laser of about $1 \mathrm{meV}$. The laser
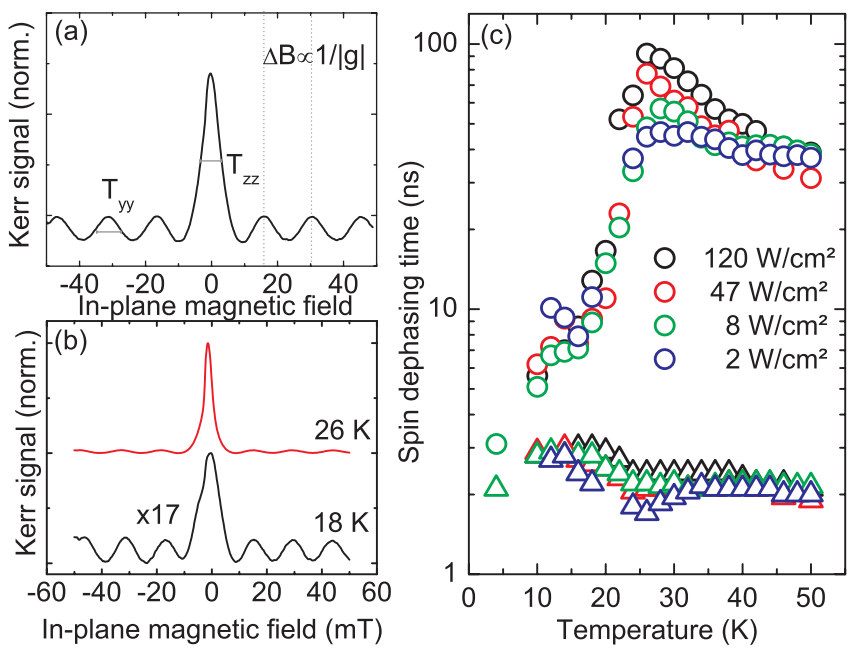

FIG. 2. (Color) (a) Typical RSA trace measured on a highmobility 2DES grown in the [110] direction. The influence of the trace features on sample parameters is indicated. (b) RSA traces measured at 18 and $26 \mathrm{~K}$. The data have been normalized and shifted. (c) Out-of plane ( $T_{z z}$, open circles) and in-plane ( $T_{y y}$, open triangles) spin dephasing times for different excitation densities as a function of temperature.

energy is kept fixed throughout the temperature-dependent and illumination-dependent measurement series. Pump and probe beams are focused to a spot size of about $50 \mu \mathrm{m}$ on the sample using an achromat. For the RSA measurements, the delay between pump and probe is kept fixed and adjusted such that the probe pulse arrives about 50 ps before the subsequent pump pulse, and the Kerr signal is recorded as a function of the applied in-plane magnetic field. For PL measurements, the pulsed Ti:sapphire laser system is detuned to higher energies to nonresonantly excite electron-hole pairs in the QW. An excitation density of about $0.2 \mathrm{~W} / \mathrm{cm}^{2}$ is used for the PL measurement series. During some of the measurements, an additional, above-barrier illumination of the sample is realized using a green $(532 \mathrm{~nm})$ continuous wave (cw) laser. The green laser is weakly focused to a spot size of about $1 \mathrm{~cm}^{2}$, which covers the whole sample, to ensure that the above-barrier illumination is homogeneous throughout the sample area probed by the Ti:sapphire laser system.

First, we discuss the shape of the RSA traces observed in our sample, outline the model, and demonstrate how all the relevant spin dynamics parameters can be extracted. Figure 2(a) shows a typical RSA trace measured on our sample. The signal contains a series of peaks corresponding to the commensurability of the spin precession period in the external field and the pump pulse repetition period $T_{\text {rep }}=12 \mathrm{~ns}$. The peak width and height are related with the spin relaxation rates. ${ }^{30}$ We clearly see that the RSA peak centered around zero magnetic field is more pronounced than the RSA peaks for finite fields, whose heights and widths are equal in the magnetic-field range investigated. This trace shape is direct evidence of the specific spin-orbit field symmetry in almost symmetric (110)-oriented systems, which is predominantly oriented along the sample growth axis $z \|$ [110] (Dresselhaus field). It leads to fast DP spin relaxation of in-plane spin 
components and slow relaxation of the $z$ spin component due to either regular or random Rashba fields. ${ }^{31,32}$ Indeed, for long spin relaxation time $T_{z z}$, the $z$ spin component is efficiently accumulated at zero magnetic field $B$, resulting in constructive interference of spins created by the train of pump pulses, giving rise to a large Kerr signal at negative time delays. For applied in-plane magnetic fields, the optically oriented electron spins precess into the sample plane, and therefore more rapid dephasing due to the DP mechanism occurs. However, some spin polarization remains within the sample during the time between subsequent pump pulses, and if the Larmor precession frequency is commensurate with the laser repetition rate, constructive interference occurs, resulting in weaker and broader maxima.

\section{THEORETICAL APPROACH}

To obtain a quantitative description of RSA traces, we follow Ref. 30 and derive the following expression for the spin $z$ component as a function of $B$ and pump-probe time delay $(\Delta t<0)$ :

$$
\frac{s_{z}(\Delta t)}{s_{0}}=e^{-\left(T_{\text {rep }}+\Delta t\right) / \bar{T}} \frac{e^{T_{\text {rep }} / \bar{T}} \mathcal{C}\left[\tilde{\Omega}\left(T_{\text {rep }}+\Delta t\right)\right]-\mathcal{C}(\tilde{\Omega} \Delta t)}{2\left[\cosh \left(T_{\text {rep }} / \bar{T}\right)-\cos \left(\tilde{\Omega} T_{\text {rep }}\right)\right]},
$$

where $s_{0}$ is the spin injected by a single pump pulse, $\bar{T}^{-1}=$ $\left(\Gamma_{y y}+\Gamma_{z z}\right) / 2$, the function

$$
\mathcal{C}(\xi)=\cos \xi+\left[\left(\Gamma_{y y}-\Gamma_{z z}\right) / 2 \tilde{\Omega}\right] \sin \xi,
$$

$\Gamma_{i j}$ [with $\left.i, j=x, y, z(x\|[1 \overline{1} 0], y\|[00 \overline{1}])\right]$ are the spin relaxation rate tensor components, and $\tilde{\Omega}=\sqrt{\left(g \mu_{\mathrm{B}} B / \hbar\right)^{2}-\Gamma_{y y}^{2} / 4}$ is the electron spin precession frequency, with $g$ being the electron Landé factor and $\mu_{\mathrm{B}}$ being the Bohr magneton. Equation (1) is derived under the assumption that $z$ spin component relaxation is driven by the regular Rashba field, in which case the spin relaxation rate tensor is nondiagonal and the spin relaxation time $T_{z z} \approx 2 / \Gamma_{z z}$; see Ref. 33 for details. If spin relaxation is determined by random Rashba fields, one has $T_{z z}=1 / \Gamma_{z z}$ and $\tilde{\Omega}=\sqrt{\left(g \mu_{\mathrm{B}} B / \hbar\right)^{2}-\left(\Gamma_{y y}-\Gamma_{z z}\right)^{2} / 4}$ in Eq. (1).

It follows from Eq. (1) that $T_{z z}$, the growth-axis SDT, is correlated with the full width at half-maximum (FWHM) of the zero-field RSA peak. $T_{y y}$, the in-plane SDT, is related to the finite-field RSA-peak FWHM. The spacing of the RSA peaks is inversely proportional to the electron $g$ factor. The results of experimental data fitting by Eq. (1) are presented in Fig. 2(c).

\section{RESULTS AND DISCUSSION}

\section{A. Temperature dependence of the spin dephasing}

Let us start with the discussion of the temperature dependence of the SDTs. Figure 2(b) shows normalized RSA traces measured at 18 and $26 \mathrm{~K}$. We note that the zero-field RSA peak drastically increases its amplitude at the higher temperature, while the finite-field RSA peaks remain nearly constant. The corresponding values of $T_{z z}$ and $T_{y y}$ are given in Fig. 2(c) in logarithmic scale. For the whole range of excitation densities used in our measurements, we see a drastic increase of $T_{z z}$ from below $10 \mathrm{~ns}$ at low temperatures to about $100 \mathrm{~ns}$ around $25 \mathrm{~K}$, while $T_{y y} \approx 2$ ns shows no notable temperature dependence. Additionally, we observe that $T_{z z}$ and $T_{y y}$ become larger as the excitation density is increased.

Now we consider the origin of the spin relaxation times $T_{y y}$ and $T_{z z}$ and their temperature dependence. The relaxation of the in-plane spin components is well-described by the DP mechanism resulting from the Dresselhaus SOI,

$$
H_{D}=-\gamma k_{x} \sigma_{z}\left[\left\langle k_{z}^{2}\right\rangle+\left(2 k_{y}^{2}-k_{x}^{2}\right)\right] / 2,
$$

where $\gamma$ is the bulk Dresselhaus coupling constant, $\sigma_{z}$ is the Pauli matrix, $k_{x}, k_{y}$ are in-plane components of the electron wave vector, and $\left\langle k_{z}^{2}\right\rangle \approx \pi^{2} / w^{2}$, where $w$ is the QW width. With the temperature increase, if the electron concentration remains constant, electron-electron collisions are expected to reduce the DP spin relaxation rate. ${ }^{34}$ However, in a complicated system with four remote dopant layers, a temperature-dependent electron density redistribution as well as ionization of the donors are expected. This redistribution leads to an increase in the electron concentration in the 2DES. To observe this effect, we perform temperature-dependent PL measurements. The PL of the 2DES has a characteristic, shark-fin-like shape. It stems from the recombination of electrons from the lowest-lying state in the 2DES up to the Fermi energy, with holes in the valence band. In a 2DES, the Fermi energy is proportional to the carrier density, therefore the FWHM of the PL may be used to track changes of the local carrier density under excitation conditions similar to those during the RSA measurements. As seen in Fig. 1(c), the FWHM of the PL from the 2DES increases with rising temperature. As the temperature is increased from 4 to $30 \mathrm{~K}$, the Fermi energy and corresponding electron density of the 2DES almost double. The corresponding increase in the spin precession rate due to the linear and cubic in-plane momentum contributions largely compensates for the temperature-induced decrease in the electron-electron collision time, rendering the relaxation time $T_{y y}$ weakly temperature-dependent.

The Dresselhaus term, however, does not cause relaxation of the $z$ component. There are two main origins of the low-temperature value of $T_{z z}$ of the order of 2 ns observed in the experiment [Fig. 2(c)]. First, one can expect some "frozen" asymmetry $\Delta n$ in the electron density to the left and to the right of the 2DES due to the trapping of carriers, either in the AlAs layers surrounding the doping or in the spacer layers between the remote doping and the QW. This asymmetry leads to the Rashba coupling $\alpha_{R}=2 \pi \xi e^{2} \Delta n / \kappa$, where $\xi=5 \times 10^{-2} \mathrm{~nm}^{2}$ is the Rashba coefficient for GaAs, $e$ is the electron charge, and $\kappa$ is the dielectric constant. Using the experimental data demonstrating that at $4 \mathrm{~K}, T_{z z} \approx T_{y y}$ and assuming the same DP relaxation mechanism for all spin components, we obtain the condition $\alpha_{R} \approx \gamma\left\langle k_{z}^{2}\right\rangle / 2$. Taking into account a broad spread in experimentally reported values of $\gamma$ from 5 to $28 \mathrm{eV} \AA^{3}$ (Refs. 35-41), one can estimate that the required asymmetry $\Delta n$ lies in the range between $\sim 0.5 \times 10^{11}$ and $\sim 3 \times 10^{11} \mathrm{~cm}^{-2}$. Second, the random electric field of the dopants, assuming that they are not fully screened by the charge carriers in the AlAs layers, ${ }^{32}$ leads to a random Rashba 
field and spin relaxation rate,

$$
\Gamma_{z z}=\frac{16 \pi}{\hbar^{3}} \frac{m e^{4} \xi^{2} n_{d} k_{F}}{\kappa^{2} R_{d}},
$$

where $m$ is the electron effective mass, $n_{d}$ is the donor concentration per one side of the 2DES, $R_{d}$ is the distance from the 2DES to the dopant layer, and $k_{F}$ is the Fermi momentum. ${ }^{42}$ The nominal concentration $n_{d}$ of the order of $5 \times 10^{12} \mathrm{~cm}^{-2}$ and the distance to the 2DES $R_{d}=85 \mathrm{~nm}$ lead to the spin relaxation time of the same few ns order of magnitude. With the temperature increase, the frozen asymmetry disappears, and the charge redistribution of itinerant electrons and electrons in the vicinity of dopant layers ${ }^{27}$ switches on the screening of the random Rashba field, leading to $T_{z z}$ values of the order of 50-100 ns.

\section{B. Excitation density dependence of the spin dephasing}

Next, we focus on the influence of excitation density on the SDTs. As discussed above, both $T_{z z}$ and $T_{y y}$ increase with an increase of the excitation density, in stark contrast to previous measurements on (110)-grown $2 \mathrm{DES},{ }^{18,43}$ where increasing excitation (or probing) density led to reduction of the SDT due to spin dephasing via the BAP mechanism. To understand this difference, we need to consider the difference in the experiments: while the spin noise spectroscopy and Hanle-type experiments utilize cw illumination of a sample, the RSA technique uses a pulsed laser system. The remaining spin polarization is probed about $12 \mathrm{~ns}$ after pulsed excitation, when photocarrier relaxation and recombination taking place on a sub-ns time scale are complete. Hence, even if the spin relaxation rate is increased while photocreated holes are present in the sample, the BAP mechanism is absent for the majority of the measurement time. A trivial explanation for the increase observed for $T_{z z}$ and $T_{y y}$ with the excitation density is a reduction of the single electron momentum scattering time due to pumping-induced heating of the 2DES, which leads to an increase of the spin dephasing time in the motional-narrowing regime of the DP mechanism.

\section{Optical gating effects on the spin dephasing}

Finally, we study the influence of above-barrier illumination on the SDTs at low temperature. Figure 1(d) shows the effect of weak, above-barrier illumination on the 2DES PL: with increasing excitation density, the width of the 2DES PL peak is reduced significantly, corresponding to a partial depletion of the 2DES. This effect, which may even lead to the inversion of the carrier type from $p$ to $n$ in a $p$-modulation-doped $\mathrm{QW},{ }^{44}$ is often referred to as optical gating and stems from a redistribution of charge carriers from the 2DES to the remote dopant sites. ${ }^{45}$ This reduction of the 2 DES carrier density is directly visible in the RSA traces in Fig. 3(a): for increasing illumination intensity, both the zero-field and finite-field peaks initially become more pronounced and narrow, while they broaden again for higher intensity. Correspondingly, the extracted SDTs [Fig. 3(b)] drastically increase with illumination intensity, reaching values above $150 \mathrm{~ns}$ for $T_{z z}$ and $25 \mathrm{~ns}$ for $T_{y y}$, before decreasing again slightly for higher illumination intensity. We may attribute this large increase to several effects:

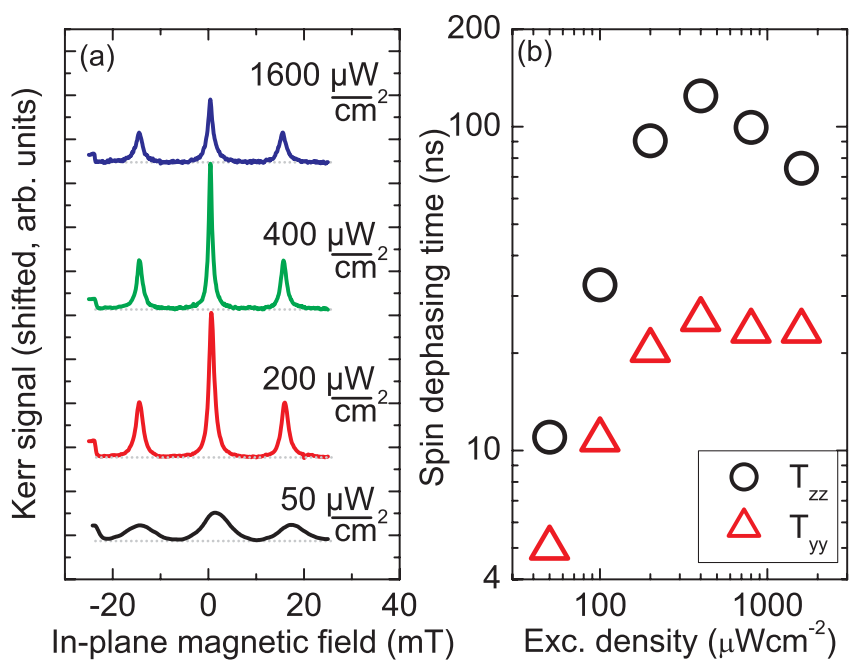

FIG. 3. (Color) (a) RSA traces measured at $4 \mathrm{~K}$ for different excitation densities of above-barrier illumination. (b) Out-of plane ( $T_{z z}$, open circles) and in-plane ( $T_{y y}$, open triangles) spin dephasing times for different excitation densities of above-barrier illumination.

the above-barrier illumination apparently symmetrizes the distribution of ionized donors, thus reducing the growth-axis electric field and the associated Rashba field. Additionally, the reduced carrier density reduces the Fermi wave vector and thus the SOI for electrons at the Fermi surface, slowing down spin dephasing due to the DP mechanism, as well as reducing the single-electron momentum relaxation time due to decreased Coulomb screening and increased electron-electron scattering rate. ${ }^{34}$ It is noteworthy that even at the lowest possible electron densities, we do not observe any decrease of the RSA peak amplitude with increasing magnetic field, which rules out spin dephasing of localized electrons. Therefore, we may infer that the observed decrease of the SDTs for high above-barrier illumination intensity stems from the BAP mechanism, which becomes relevant due to the increased hole density in the QW.

\section{CONCLUSION}

In conclusion, we have investigated the spin dephasing in a high-mobility (110)-grown two-dimensional electron system by resonant spin amplification measurements. We observe a strong anisotropy of the SDTs for in- and outof-plane spin orientation, as well as a strong temperature dependence of the out-of-plane SDT, which we attribute to dopant-ionization-related changes in the growth-axis electric field. For weak above-barrier illumination, SDTs above 150 ns are reached at low temperatures for delocalized carriers, exceeding previously reported values for (110)-grown 2DES by an order of magnitude.

\section{ACKNOWLEDGMENTS}

The authors would like to thank D. R. Yakovlev and S. A. Tarasenko for fruitful discussion. Financial support by the DFG via SFB689 and SPP 1285, RFBR, RF President Grant NSh-5442.2012.2, MCINN of Spain (Grant No. FIS200912773-C02-01), and Basque Country Government (Grant No. IT-472-10) is gratefully acknowledged. 
*glazov@coherent.ioffe.ru

† tobias.korn@physik.uni-regensburg.de

${ }^{1}$ I. Zutic, J. Fabian, and S. Sarma, Rev. Mod. Phys. 76, 323 (2004).

${ }^{2}$ M. W. Wu, J. H. Jiang, and M. Q. Weng, Phys. Rep. 493, 61 (2010).

${ }^{3}$ O. Z. Karimov, G. H. John, R. T. Harley, W. H. Lau, M. E. Flatte, M. Henini, and R. Airey, Phys. Rev. Lett. 91, 246601 (2003).

${ }^{4}$ Y. A. Bychkov and E. I. Rashba, J. Phys. C 17, 6039 (1984).

${ }^{5}$ M. Dyakonov and V. Perel, Sov. Phys. JETP 33, 1053 (1971).

${ }^{6}$ M. Dyakonov and V. Kachorovskii, Sov. Phys. Semicond. 20, 110 (1986).

${ }^{7}$ Y. Ohno, R. Terauchi, T. Adachi, F. Matsukura, and H. Ohno, Phys. Rev. Lett. 83, 4196 (1999).

${ }^{8}$ S. Döhrmann, D. Hägele, J. Rudolph, M. Bichler, D. Schuh, and M. Oestreich, Phys. Rev. Lett. 93, 147405 (2004).

${ }^{9}$ N. S. Averkiev and L. E. Golub, Phys. Rev. B 60, 15582 (1999).

${ }^{10}$ B. Liu, H. Zhao, J. Wang, L. Liu, W. Wang, D. Chen, and H. Zhu, Appl. Phys. Lett. 90, 112111 (2007).

${ }^{11}$ D. Stich, J. H. Jiang, T. Korn, R. Schulz, D. Schuh, W. Wegscheider, M. W. Wu, and C. Schüller, Phys. Rev. B 76, 073309 (2007).

${ }^{12}$ T. Korn, D. Stich, R. Schulz, D. Schuh, W. Wegscheider, and C. Schüller, Physica E 40, 1542 (2008).

${ }^{13}$ T. Korn, D. Stich, R. Schulz, D. Schuh, W. Wegscheider, and C. Schüller, AIP Conf. Proc. 1199, 425 (2009).

${ }^{14}$ J. J. Baumberg, D. D. Awschalom, N. Samarth, H. Luo, and J. K. Furdyna, Phys. Rev. Lett. 72, 717 (1994).

${ }^{15}$ M. Oestreich, M. Römer, R. J. Haug, and D. Hägele, Phys. Rev. Lett. 95, 216603 (2005).

${ }^{16}$ O. D. D. Couto, F. Iikawa, J. Rudolph, R. Hey, and P. V. Santos, Phys. Rev. Lett. 98, 036603 (2007).

${ }^{17}$ G. L. Bir, A. G. Aronov, and G. E. Pikus, Sov. Phys. JETP 42, 705 (1975).

${ }^{18}$ G. M. Müller, M. Römer, D. Schuh, W. Wegscheider, J. Hübner, and M. Oestreich, Phys. Rev. Lett. 101, 206601 (2008).

${ }^{19}$ G. M. Müller, M. Oestreich, M. Römer, and J. Hübner, Physica E 43, 569 (2010).

${ }^{20}$ G. Salis, D. T. Fuchs, J. M. Kikkawa, D. D. Awschalom, Y. Ohno, and H. Ohno, Phys. Rev. Lett. 86, 2677 (2001).

${ }^{21}$ J. M. Kikkawa and D. D. Awschalom, Phys. Rev. Lett. 80, 4313 (1998).

${ }^{22}$ J. Kikkawa and D. Awschalom, Nature (London) 397, 139 (1999).

${ }^{23}$ G. V. Astakhov, M. M. Glazov, D. R. Yakovlev, E. A. Zhukov, W. Ossau, L. W. Molenkamp, and M. Bayer, Semicond. Sci. Technol. 23, 114001 (2008).
${ }^{24}$ T. Korn, M. Kugler, M. Griesbeck, R. Schulz, A. Wagner, M. Hirmer, C. Gerl, D. Schuh, W. Wegscheider, and C. Schüller, New J. Phys. 12, 043003 (2010).

${ }^{25}$ I. A. Yugova, A. A. Sokolova, D. R. Yakovlev, A. Greilich, D. Reuter, A. D. Wieck, and M. Bayer, Phys. Rev. Lett. 102, 167402 (2009).

${ }^{26}$ V. Umansky, M. Heiblum, Y. Levinson, J. Smet, J. Nübler, and M. Dolev, J. Cryst. Growth 311, 1658 (2009).

${ }^{27}$ C. Rössler, T. Feil, P. Mensch, T. Ihn, K. Ensslin, D. Schuh, and W. Wegscheider, New J. Phys. 12, 043007 (2010).

${ }^{28}$ M. Griesbeck, M. M. Glazov, T. Korn, E. Y. Sherman, D. Waller, C. Reichl, D. Schuh, W. Wegscheider, and C. Schüller, Phys. Rev. B 80, 241314 (2009).

${ }^{29}$ T. Korn, Phys. Rep. 494, 415 (2010).

${ }^{30}$ M. M. Glazov and E. L. Ivchenko, Semiconductors 42, 951 (2008).

${ }^{31}$ M. M. Glazov, E. Y. Sherman, and V. K. Dugaev, Physica E 42, 2157 (2010).

${ }^{32}$ M. M. Glazov, M. A. Semina, and E. Y. Sherman, Phys. Rev. B 81, 115332 (2010)

${ }^{33}$ S. A. Tarasenko, Phys. Rev. B 80, 165317 (2009).

${ }^{34}$ M. M. Glazov and E. L. Ivchenko, JETP 99, 1279 (2004).

${ }^{35}$ G. Pikus, V. Maruschak, and A. Titkov, Sov. Phys. Semicond. 22, 115 (1988).

${ }^{36}$ B. Jusserand, D. Richards, G. Allan, C. Priester, and B. Etienne, Phys. Rev. B 51, 4707 (1995).

${ }^{37}$ J. B. Miller, D. M. Zumbühl, C. M. Marcus, Y. B. Lyanda-Geller, D. Goldhaber-Gordon, K. Campman, and A. C. Gossard, Phys. Rev. Lett. 90, 076807 (2003).

${ }^{38} \mathrm{R}$. Winkler, Spin-Orbit Coupling Effects in Two-Dimensional Electron and Hole Systems (Springer, Berlin, 2003).

${ }^{39}$ W. J. H. Leyland, R. T. Harley, M. Henini, A. J. Shields, I. Farrer, and D. A. Ritchie, Phys. Rev. B 76, 195305 (2007).

${ }^{40}$ J. D. Koralek, C. P. Weber, J. Orenstein, B. A. Bernevig, S.-C. Zhang, S. Mack, and D. D. Awschalom, Nature (London) 458, 610 (2009).

${ }^{41}$ A. Balocchi, Q. H. Duong, P. Renucci, B. L. Liu, C. Fontaine, T. Amand, D. Lagarde, and X. Marie, Phys. Rev. Lett. 107, 136604 (2011).

${ }^{42}$ E. Y. Sherman, Appl. Phys. Lett. 82, 209 (2003).

${ }^{43}$ R. Völkl, M. Griesbeck, S. A. Tarasenko, D. Schuh, W. Wegscheider, C. Schüller, and T. Korn, Phys. Rev. B 83, 241306 (2011).

${ }^{44}$ M. Syperek, D. R. Yakovlev, A. Greilich, J. Misiewicz, M. Bayer, D. Reuter, and A. D. Wieck, Phys. Rev. Lett. 99, 187401 (2007).

${ }^{45}$ A. Chaves, A. Penna, J. Worlock, G. Weimann, and W. Schlapp, Surf. Sci. 170, 618 (1986). 